\title{
Participación social: Planteamiento y evaluación de un modelo estructural a partir de variables observadas.
}

\author{
JOSE SABARIEGOS DIAZ \\ UNIVERSIDAD DE ALICANTE
}

Resumen: Una de las cuestiones significativas de la sociología es la participación social. Tal participación se lleva a cabo a través de la interacción entre personas con un objetivo en común. Lo importante no es conocer ese objetivo sino los impulsos que llevan a cada una de las personas a integrarse en un conjunto. Estos impulsos a los que llamaremos variables, estarán relacionados entre sí a veces de manera directa, y otras veces de manera indirecta. En el presente trabajo se tratará de explicar la participación social a través de unas variables escogidas para tal efecto. Tales variables objeto del estudio serán la educación, la clase social, la religión y la ideología.

Palabras clave: educación universitaria; clase social; actitud religiosa; ideología; participación social.

\begin{abstract}
The social involvement is one of the most important issues in sociology. Such involvement is carried out by the interaction among people who have common aims. The important thing is not to be familiar with this aim but to be familiar with the boosts which cause that every single person fit into a group. These boosts will be called variable and they are sometimes tied together in a direct way and other times in an indirect way. In the current work, we are going to explain the social involvement by means of selected variables for such purpose.
\end{abstract}

The education, the social class, the religion and the ideology will be the variables object of study.

Key words: university education; social class; religious attitude; ideology; social involvement. 


\section{Introducción}

Las personas, con frecuencia, se asocian para realizar actividades que de otro modo no las llevarían a cabo por ellas mismas, y uno de los principales modos de conseguir esas acciones cooperativas es a través de la organización donde las acciones colectivas son realizadas para alcanzar un objetivo común (Aldrich y Marsden, 1988).

Según Putnam (1993), 'la democracia prospera cuando existe un fuerte capital social', por lo que hay relación entre los compromisos políticos, el asociacionismo y otras formas de compromiso cívico y social. Si la participación social disminuye, también lo hará la participación democrática, y en consecuencia, la ideología iría perdiendo su importancia en la sociedad a favor de otros valores.

Para concretar lo que llamamos capital social hay que recurrir a una de las primeras definiciones generalistas de Teorell quien lo define como las distintas facetas de las redes sociales que vinculan a la gente con su entorno social' (Teorell, 2000)

Llegamos entonces al objetivo de nuestro trabajo, que no es otro que el de encontrar las relaciones que existen entre las distintas facetas del entorno social de las personas y que impulsan a estas a la participación social.

La participación social es una de las más grandes expresiones del compromiso cívico que puede ser definida como 'la interacción de unos individuos con otros en situaciones y procesos más o menos estructurados, y que desarrollan una actividad con fines significativos dentro del sistema social' (Francés, 2005).

Esta definición plantea algunas cuestiones que se irán desarrollando a lo largo del trabajo y que ponen énfasis en la estructura final del modelo a tratar.

De base se plantea que tanto la educación, la clase social, la religión o la ideología son procesos, estados, situaciones o ideas que están relacionados con la participación social, es decir, que directa o indirectamente tienen que ver con el hecho participativo o con el impulso que lleva a una persona a participar dentro de una asociación, organización o colectivo grupal.

García-Alandete y Pérez Delgado demostraban la existencia de relación entre la religiosidad y los valores individuales ${ }^{6}$, es decir, según el grado de religiosidad, a nivel individual afectarán unos valores u otros que a su vez estarán relacionados con el hecho de participar socialmente en cualquier organización.

Cuando en estas relaciones se introduce la educación, y más concretamente educación universitaria, vemos como son estos alumnos los que obtienen unos valores susceptibles de provocar el hecho participativo. En este sentido, se decantan por cuestiones no materiales y participativas ${ }^{7}$.

El planteamiento teórico que se propone para elaborar dicho trabajo tiene que ver con las variables citadas anteriormente. En este sentido se propone que: la educación, la religión, la clase social y la ideología son variables que pueden explicar la participación social.

\footnotetext{
${ }^{6}$ Actitudes religiosas y valores de un grupo de jóvenes universitarios españoles. En bibliografía.

${ }^{7}$ Investigación sobre los valores de los jóvenes universitarios españoles. En bibliografía.
} 
Ya hemos visto como existe una relación entre la religiosidad y los valores individuales que pueden impulsar el hecho participativo, y cómo son los estudiantes universitarios los más propensos a ser influidos por este tipo de valores.

En relación a la ideología, Tinoco Amador $^{8}$ demuestra la relación que existe entre esta y la religión. En éste sentido se puede observar que los niveles más altos de religiosidad se obtienen en aquellas opciones políticas de derechas, sin embargo, no ocurre así con los índices más bajos de religiosidad que corresponden con las teorías políticas más progresistas.

René Bendit ${ }^{9}$ aprovecha la ideología para relacionarla con la participación social, y en este sentido demuestra que 'el autoposicionamiento ideológico y político es un factor diferenciador en cuanto a las causas más movilizadoras' (INJUVE, 1995: 63). Propone que son los posicionamientos de izquierdas los que se implican en prestar una mayor atención a los problemas sociales y los que tienden a movilizarse en forma de organizaciones, asociaciones, etc.

\section{Metodología e Hipótesis Causales}

Una explicación para las motivaciones que impulsan a participar en cualquier organización social es el hecho de considerar un modo de vida determinado como susceptible a ser considerado el adecuado para proporcionar al individuo la satisfacción plena de participar en un acontecimiento social.

Este modo de vida particular viene establecido por las variables anteriormente citadas. De esta manera podemos establecer las siguientes hipótesis:

- Adquirir una educación universitaria determina en parte el grado de religiosidad. En este caso, se cree que una educación universitaria afecta negativamente a la fe de una religión.

- La clase social a la que se pertenezca influirá tanto al nivel de religiosidad como a la ideología individual. En este caso, una clase social alta implicará una fe religiosa y una ideología tendente a la derecha.

- La religión determinará el carácter ideológico, es decir, tienen una relación explicativa. En este caso, tener fe estaría relacionado con una ideología orientada a la derecha, y una ausencia de fe estaría relacionado con una ideología de izquierdas.

- La ideología determinará si una persona es partidaria o no de la participación social. Se cree que una ideología de izquierdas es más proclive a favorecer la participación social que una ideología de derechas.

Tanto la educación como la clase social o la religión explican el hecho participativo de una forma indirecta, aunque no lo hace así la ideología que lo explica de una forma directa. Pero vamos a definir cada una de las variables explicando las características de su análisis. Llamaremos educación exclusivamente a aquella conseguida en el ámbito universitario, es decir, en el modelo estructural desarrollado analizaremos a individuos universitarios y no

\footnotetext{
${ }^{8}$ Religiosidad y preferencia política en universitarios de la ciudad de México. En bibliografía.

${ }^{9}$ Participación social y política de los jóvenes en países de la Unión Europea. En bibliografía.
} 
universitarios, cuyas relaciones con otras variables se alterarán si se obtiene o no una educación universitaria.

La clase social se ha dividido en dos grupos englobando a aquellos que se consideraban dentro de una clase social alta y los considerados de la clase social baja.

La religión viene expresada a través de aquellos que se han considerados religiosos y los que se han considerado no religiosos.

La ideología se ha dividido al igual que la clase social en dos grupos diferenciados. Entre aquellos que se han considerado defensores de una política de izquierdas y los que apoyan ideologías de derechas.

En el caso de la participación social sólo se ha considerado el hecho de cumplir esta acción, ya sea en el ámbito religioso, juvenil, deportivo, político, etc. Se tratará la variable en función de si se participa o no en organizaciones sociales sean del ámbito que sean.

Para desarrollar dicho trabajo se ha recurrido a las bases estadísticas del International Social Survey Programme (ISSP ${ }^{10}$ ) utilizando los datos elaborados del cuestionario Social Networks del año 2001. La elección de determinadas variables para su posterior procesamiento ha dado lugar a un modelo estructural que explicaremos a continuación.

El modelo estructural viene determinado por un orden causal cuyas variables van determinando otras hasta dar con la explicación deseada. La causalidad viene determinada por la relación existente entre unas y otras variables siempre que exista covariación entre ellas y sean susceptibles de una relación causa-efecto.

El modelo estructural, cuya finalidad es la de determinar relaciones explicativas, parte de dos variables exógenas que como tales, no pueden ser explicadas. Las variables a las que nos referimos son la educación y la clase social. La primera de ellas tiene una relación causaefecto directa respecto a la variable religión, y una relación indirecta respecto a la variable ideología.

En el caso de la otra variable exógena, la clase social, esta tiene relación directa con las variables religión e ideología, y con esta última también posee una expresión conjunta de efectos directos e indirectos.

También hay que señalar la relación causa-efecto directa que la variable religión posee con la ideología, e indirecto con la participación social, siendo la ideología la única variable del modelo que posee una relación directa con la variable a explicar, la participación social.

\section{Resultados}

En el anterior apartado hemos visto como quedaba formulado un modelo en el que la participación social venía explicada por distintas variables sociodemográficas que, covariando entre sí, establecían una relación con la variable a explicar.

${ }^{10}$ ISSP Programa Internacional de Encuestas Sociales. www.issp.org 
Para ajustar el modelo propuesto se ha trabajado con la herramienta estadística LISREL utilizada frecuentemente para analizar las relaciones de dependencia que se establecen entre las variables propias de un modelo estructural y que influyen en un proceso social. Pero antes de explicar con detalle las relaciones que este programa nos ofrece hemos de representar dicho diagrama para entender las posteriores relaciones, así como la matriz de efectos, la densidad del propio modelo y sus ecuaciones estructurales.

\section{Diagrama Causal Del Modelo}

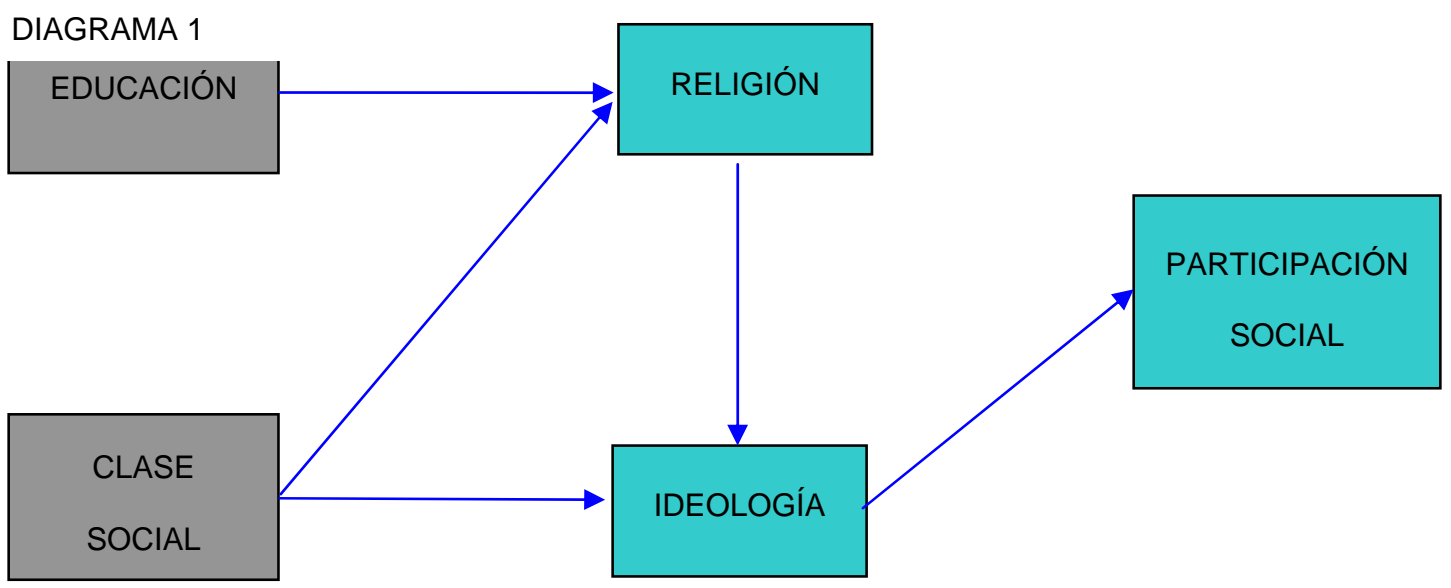

MATRIZ DE EFECTOS

\begin{tabular}{l|lllll} 
& RELIGIÓN & IDEOLOGÍA & $\begin{array}{l}\text { PART. } \\
\text { SOCIAL }\end{array}$ & EDUCACIÓN & $\begin{array}{l}\text { CLAS. } \\
\text { SOCIAL }\end{array}$ \\
\hline RELIGIÓN & - & 0 & 0 & 1 & 1 \\
IDEOLOGÍA & 1 & - & 0 & 0 & 1 \\
PART. & 0 & 1 & - & 0 & 0 \\
SOCIAL & & & & &
\end{tabular}

\section{Densidad Del Modelo}

Podríamos definir la 'densidad' como el nivel general de conexión entre los puntos de un grafo en tanto que depende de la inclusividad del propio grafo como de la suma de los grados de sus puntos. Pero cuando entran en juego variables exógenas, la densidad ${ }^{11}$ hemos de calcularla a partir de las relaciones existentes y de las relaciones posibles en el modelo.

${ }^{11}$ Densidad en modelos estructurales con variables exógenas. Representada con apostrofe para diferenciarla del resto de densidades. 


$$
\text { Densidad' }=\frac{\text { l.existentes }}{n *(n-1)+n * m}
$$

De la anterior fórmula obtenemos que:

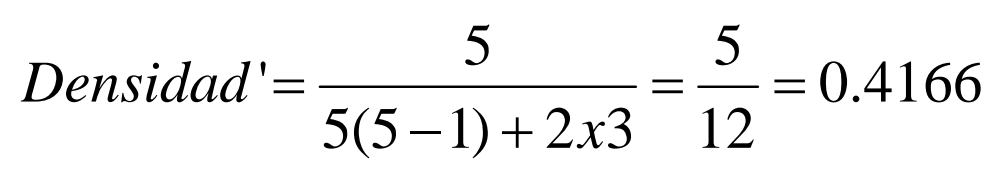

Por tanto, la densidad entre las variables es de $41.66 \%$.

ECUACIONES ESTRUCTURALES

$\underline{\text { Ideología }}=0.20 *$ Religión $+0.24 *$ Clase Social, Errorvar. $=0.24, \quad$ Rý $=0.048$

$\begin{array}{lcc}(0.039) & (0.098) & (0.013) \\ 5.08 & 2.46 & 18.45\end{array}$

$\underline{\text { Religión }}=-0.15 *$ Educación $+0.28 *$ Clase Social, Errorvar. $=0.2, \quad$ Rý $=0.018$
$(0.060)$
$-2.57$
$(0.097)$
2.93
$(0.012)$
18.45

$\underline{\text { Participación Social }}=-0.081 *$ Ideología, Errorvar. $=0.19, \quad$ Rý $=0.0083$
$(0.034)$
(0.011)

$-2.39$

18.45 


\section{Testado Y Validación Del Modelo}

AJUSTE DEL MODELO ESTRUCTURAL

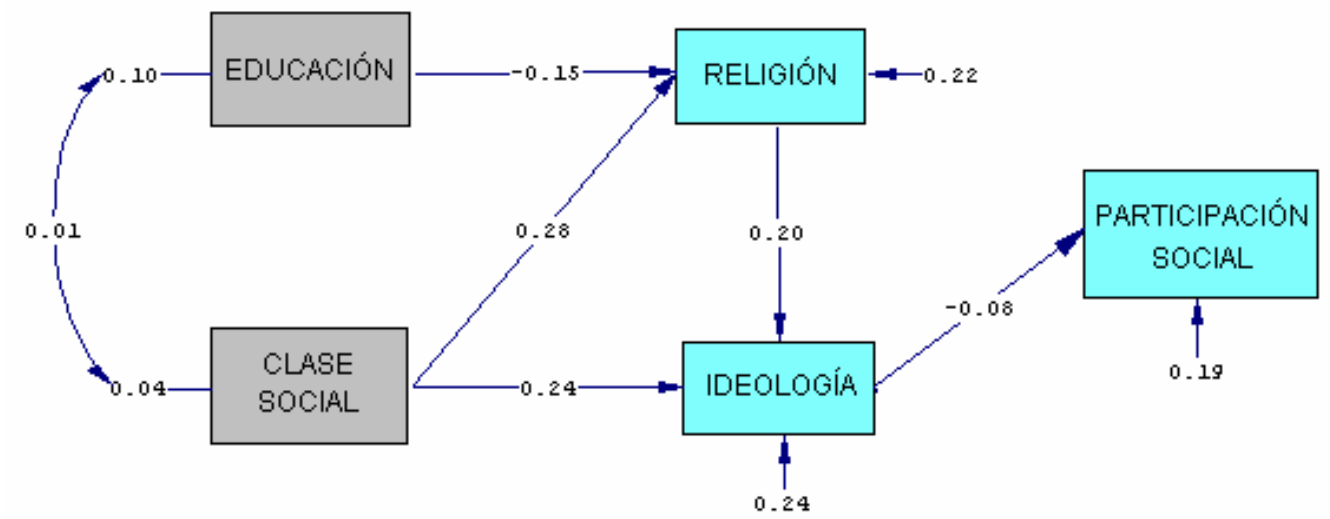

Chi-Square $=3.11, \mathrm{df}=4$, P-value $=0.53977, \mathrm{RMSEA}=0.000$

Tal y como podemos observar en la solución estimada que nos presenta el programa LISREL en el anterior diagrama, las relaciones de dependencia que se establecen entre las variables resultan ser consistentes. De hecho, tal y como se verá a continuación, los $t$-V alues son significativos distintos a 0 ya que ninguno de ellos se encuentra entre los valores de $1.96 \mathrm{y}+1.96$.

En el diagrama podemos observar como la variable 'ideología' soporta cargas de la variable 'clase social', y en menor medida de 'religión' ( 0.24 y 0.20 respectivamente). En el caso de la otra variable endógena interviniente, la variable 'religión', ésta soporta cargas de las variables exógenas, tanto de 'educación' (-0.15) como de 'clase social (0.28). En el caso de la variable endógena final que queremos explicar, 'participación social', ésta recibe únicamente carga de la variable 'ideología' (-0.08).

El siguiente diagrama del modelo desarrollado a partir del programa LISREL representa los t-values para cada parámetro estimado. Este diagrama nos servirá a modo de comprobación de que los valores de los t-values son significantes.

DIAGRAMA 3

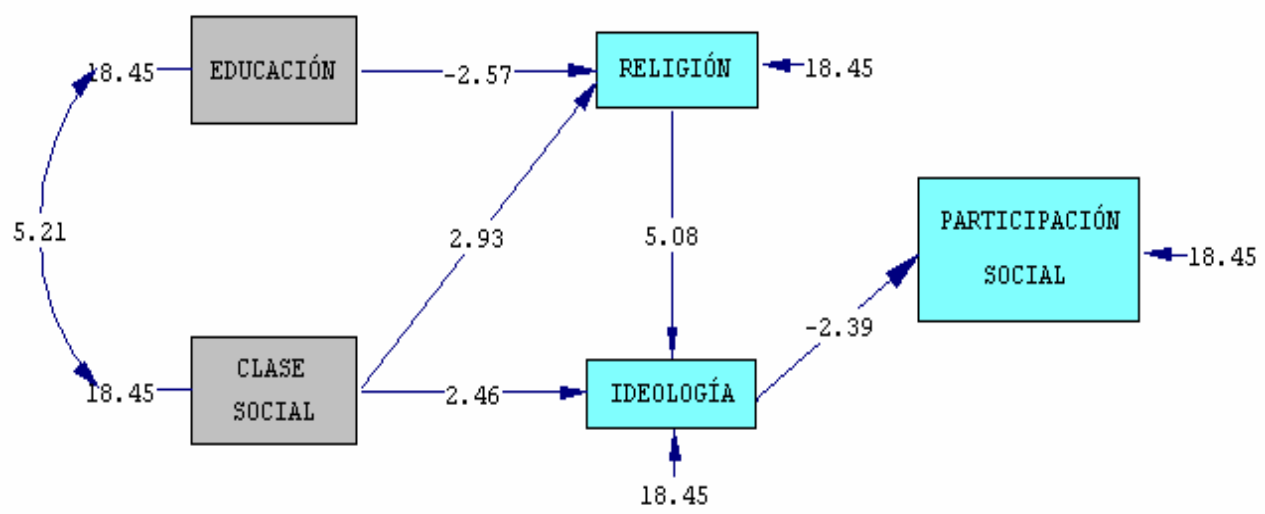

Chi-Square $=3.11, d f=4, P$-value $=0.53977$, RMSEA $=0.000$ 


\section{ESTADÍSTICOS DE BONDAD DE AJUSTE}

\begin{tabular}{|c|}
\hline 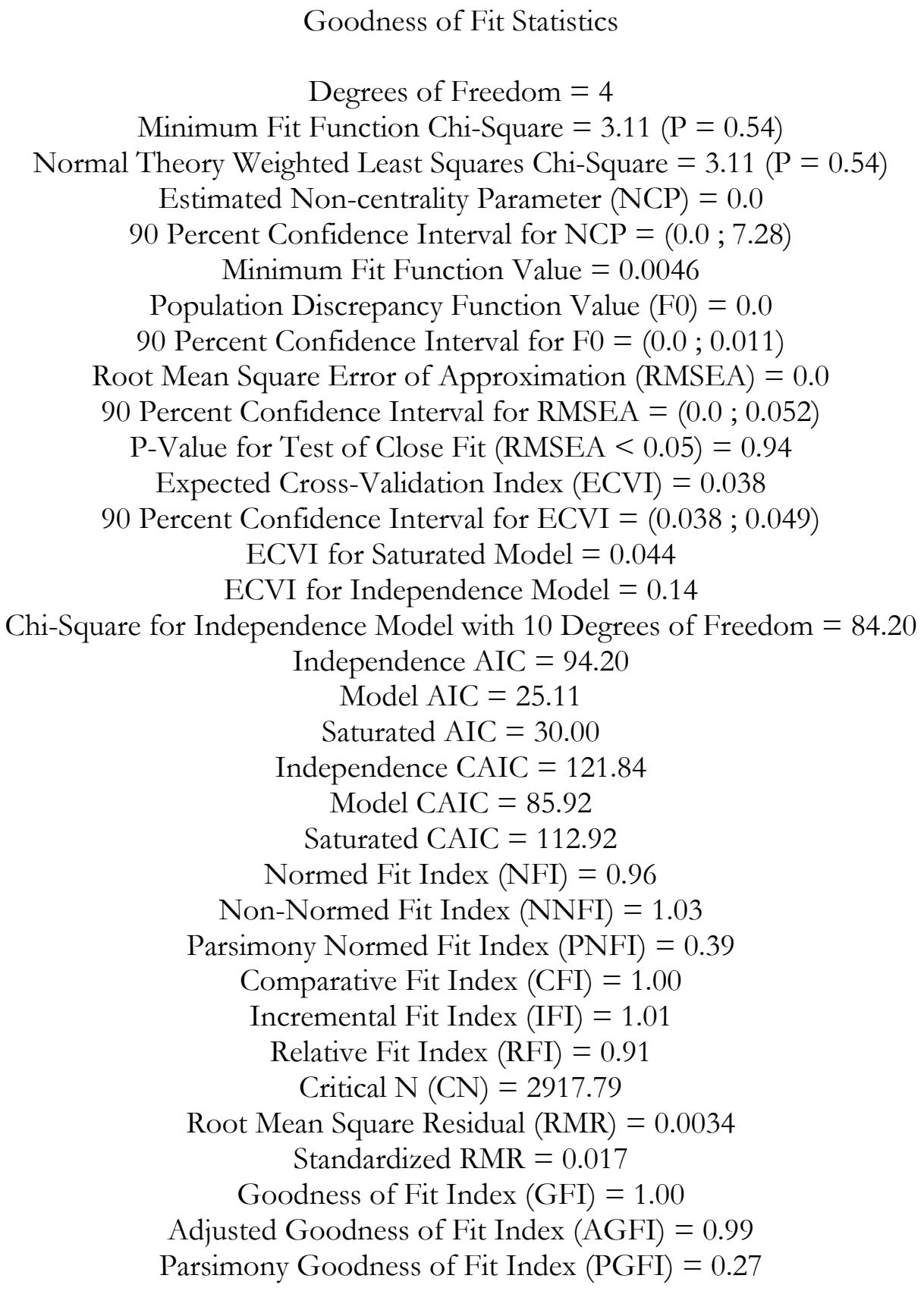 \\
\hline
\end{tabular}

Los índices más frecuentes para la validación de modelos son la Chi-square, los grados de libertad (negrees of freedom; df), el P-value y el RMSEA (Root Mean Square Error of Aproximation).

En estas pruebas de ajuste, el Chi-square nos ofrece un test de significación para la hipótesis de que las variables observadas son mutuamente independientes. En este caso, la Chi-square es bastante baja (3.11) pero se halla ajustada a los grados de libertad (4), lo que indica que se trata de un buen ajuste. En el caso del P-value, este debe tener una variación entre 0 y 1 , pero se recomienda que el valor sea mayor a 0.05 . En nuestro ajuste, este valor es de 0.53 . Y en el caso del RMSE $A$, este debe situarse entre 0.05 y 0.08 para que el ajuste 
del modelo sea moderado, y menor a 0.05 para obtener un buen ajuste. En nuestro caso, el valor es de 0.00 , por lo que el modelo propuesto ajusta correctamente sobre los datos.

A continuación, vamos a analizar varios índices de bondad de ajuste.

El llamado GFI (Goodness of Fit Index) ha obtenido el valor de 1.00, lo que supone un ajuste perfecto dentro de los parámetros recomendados.

El RMR (Root Mean Square Residual) y el Standardized RMR indican la proximidad entre la matriz de covarianzas original y la reproducida por el modelo propuesto. En nuestro caso, el valor es de 0.0034 y 0.017 respectivamente. Aunque este coeficiente es susceptible de la decisión del investigador, cuanto más cerca esté el valor de 0.00 , los residuales también estarán próximos a 0 .

Si queremos tener en cuenta los grados de libertad hemos de analizar el AGFI (Adjusted Goodness of Fit Index), donde valores próximos a 0.00 serán propios de un mal ajuste y valores próximos a 1.00 expresarán un ajuste excelente. En nuestro caso, el valor es de 0.99 , por lo que representa un ajuste bastante bueno.

Existen tres indicadores de ajuste, el NFI (Normed Fit Index), el NNFI (Non-Normed Fit Index), y el CFI (Comparative Fit Index) que están basados en los valores de la función de ajuste. Estos índices han de ser mayores de 0.9 para expresar un modelo con un ajuste adecuado. En nuestro caso, los valores obtenidos han sido 0.96, 1.03 y 1.00 respectivamente, por lo que demuestran nuevamente que el ajuste ha sido correcto.

Los índices PNFI (Parsimony Normed Fit Index) y PGFI (Parsimony Goodness of Fit Index) comparan los valores en modelos alternativos. En este caso, los valores más próximos a 1.00 tendrían un ajuste perfecto y los valores cercanos a 0.00 quedarían sin ajuste. En nuestro caso, estos valores han sido menores de 0.5, concretamente de 0.39 y 0.27 respectivamente, por lo que no llegan a establecer un ajuste perfecto pero tampoco se quedan excluidos del ajuste.

\section{Discusión y conclusiones}

El funcionamiento del modelo y su consistencia nos lleva a pensar que existe una relación entre las variables que hemos seleccionado para el trabajo.

Tal y como hemos previsto en las hipótesis, la ideología puede explicar la participación social. Pero para llegar a esta conclusión hace falta explicar primero el orden causal del modelo.

La relación entre la educación y la religión es negativa tal y como se puede observar en el diagrama 2. Esto viene a indicar que aquella población que carece de un título universitario es más proclive a creer en alguna fe, sin embargo, esa fe no aparece tanto en aquella población con conocimientos y estudios universitarios. García-Alandete y Pérez Delgado hablan en este sentido. Ellos demuestran que la población universitaria es más proclive a no seguir las doctrinas de una fe religiosa como lo puedan hacer aquellos que no tienen una educación universitaria.

En relación a la ideología, queda demostrado que existe una relación significativa y positiva entre ésta variable y la religión, de tal forma que, son aquellos seguidores de fe los que defienden una ideología con tendencia hacia la derecha. En este sentido, Tinoco Amador 
esclarece que son aquellas ideologías de izquierdas las menos relacionadas con la fe religiosa y las más con la participación social.

Con la clase social ocurre algo parecido. Hemos visto como una clase social alta está más relacionada con la religión y una ideología de derechas que una clase baja, la cual es más susceptible de desarrollar ideologías de izquierdas sin adentrarse en el mundo religioso.

De esta manera, podemos decir que es una clase social baja y una ausencia de fe religiosa lo que puede explicar una ideología de izquierdas que, según Tinoco Amador, es más susceptible de participar en acontecimientos u organizaciones sociales.

Pero esto no queda ahí. René Bendit ha relacionado la ideología con la participación social demostrando que el autoposicionamiento ideológico suele ser un factor diferenciador en cuanto a las causas más movilizadoras, planteando que son los posicionamientos de izquierdas los que más se implican en prestar una mayor atención a los problemas sociales y los que tienden a movilizarse en forma de organizaciones, actos sociales, asociaciones, etc.

En este trabajo hemos podido confirmar el planteamiento de Bendit al comprobar la existencia de relación entre estas dos variables. De tal forma que se puede considerar una relación negativa tal y cómo observamos en el diagrama 2. Esto nos indica que, efectivamente, son aquellas ideologías de izquierda las que pueden llegar a explicar la participación social.

Por tanto, podemos concluir que la tan necesaria participación social tiene relación con un perfil social determinado. A través de una educación universitaria se tiende hacia una menor fe que tiene relación con una ideología de izquierdas. En este mismo sentido, una clase social baja explica una ideología de izquierdas al estar más concienciada de los problemas sociales. Y es esta ideología junto con el empuje de la educación, la clase social y la ausencia de fe religiosa las que pueden llevar a una persona a participar en actos de carácter social.

Quedan aún muchos caminos a través de los cuales se puede enriquecer este análisis. Aspectos tales como el sexo o la situación laboral pueden favorecer a la comprensión de dicho modelo. Aun así, hemos podido observar que variables tan íntimas como la ideología o la fe pueden tener relación con la participación social, uno de los hechos sociales más importantes para la sociedad. 


\section{Referencias Bibliográficas}

Alaminos, A., Francés, F y Santacreu, O. Reflexiones teóricas y modelos empiricos sobre identidad, diversidad y participación social. Alicante. Limencop.

Alaminos, A., Francés, F Y Santacreu, O. (2005) Socialización, ideología y participación. Alicante. Limencop.

Alaminos, A. (2005) El análisis de la realidad social. Modelos estructurales de covarianzas. Alicante, Limencop.

Aldrich, H. E., Y Marsden, P. V. (1998) 'Environments and Organizations' Handbook of sociology, Newbury Park, CA, Sage.

Álvarez Rodríguez, J., Rodríguez Sabiote, C. Y Lorezo Quiles, O. 'investigación sobre los valores de los jóvenes universitarios españoles. Un estudio comparado' Revista Internacional de CCSS y humanidades. SOCIOTAM. vol.27, nº 1, 2007, pp: 25-46.

Bendit, René. Conferencia 'Participación social y política de los jóvenes en países de la Unión Europea' impartida el 17.12.1999 durante la Reunión del Grupo de Trabajo "Juventúd" CALCSO.

Francés, J. Y Santacreo, O. Modelos estructurales en Investigación Social. LISREL: programación en Simplis Manual de prácticas para Técnicas de Investigación Social III. Dto. Sociología II, Universidad de Alicante.

García-Alandete, J. Y Pérez Delgado, E. 'Actitudes religiosas y valores de un grupo de jóvenes universitarios españoles’ Anales de psicología. vol.21, n 1, junio, 2005. pp: 149-169.

INJUVE (comp.) (1995) La solidaridad de la juventud en España, Madrid.

ISSP (2001) Social Networks II. Cuestionario para España del Programa Internacional de Encuestas Sociales.

Putnam, R. (1993) 'Bowling Alone: America's Declining Social Capital'. Journal od Femocracy, $\mathrm{n}^{\circ} 6$.

Teorell, J. (2000) A resource model f social capital: Networks, recruitment and political participation in Sweden, Paper prepared for the workshop Voluntary associations, social capital and interest mediation: forging the linf, ECPR join sessions. Copenhagen.

Tinoco Amador, J. 'Religiosidad y preferencia política en universitarios de la ciudad de México’ Liberabit. Septiembre, 2006. pp: 123-134. 